\title{
An algorithm for the calculation of the partial wave expansion of the Coulomb-distorted plane wave
}

\author{
I. Hornyak ${ }^{\mathrm{a}, \mathrm{b}}$, A.T. Kruppa ${ }^{\mathrm{a}, *}$ \\ ${ }^{a}$ Institute of Nuclear Research, Bem tér 18/c, 4026 Debrecen, Hungary \\ ${ }^{b}$ University of Debrecen, Faculty of Informatics, PO Box 12, 4010 Debrecen, Hungary
}

\begin{abstract}
The partial wave expansion of the Coulomb-distorted plane wave is determined by the help of the complex generalized hypergeometric function ${ }_{2} F_{2}(a, a ; a+l+1, a-l ; z)$. An algorithm for the calculation of ${ }_{2} F_{2}(a, a ; a+l+1, a-l ; z)$ is created and it is implemented as a FORTRAN-90 code. The code is fast and its accuracy is 14 significant decimal digits.
\end{abstract}

Keywords: Coulomb, plane wave, Coulomb-distorted plane wave, hypergeometric function, FORTRAN

\section{Program Summary}

Authors: I. Hornyak and A.T. Kruppa

Program Title: Hyp2F2spec_v1

Journal Reference:

Catalogue identifier:

Licensing provisions: none

Programming language: FORTRAN-90

Computer: x86-64

Operating system: LINUX, MAC OS X, Windows

RAM: < $1 \mathrm{MB}$

Number of processors used: 1

Supplementary material:

Keywords: generalized hypergeometric function, 2F2, Coulomb distorted plane wave, partial wave expansion

Classification: 4. Computational Methods, 4.7 Other Functions

External routines/libraries:

Subprograms used:

Catalogue identifier of previous version:

Journal reference of previous version:

Does the new version supersede the previous version?:

Nature of problem:The partial wave expansion of the Coulomb distorted plane wave is given by a generalized hypergeometric function with special indexes. An algorithm is developed for the calculation of this function.

\footnotetext{
${ }^{*}$ Corresponding author. Tel.: +3652509232 ; fax: +3652416181 .

E-mail address: kruppa.andras@atomki.mta.hu.
} 
Solution method:Taylor-series and asymptotic expansion in both cases estimates for the remainder terms are determined. Explicit expression is also used.

Reasons for the new version:

Summary of revisions:

Restrictions:

Unusual features:

Additional comments:

Running time:Less than 0.01 seconds

\section{Introduction}

Scattering of charged particles is an important field in quantum mechanics. Even the two-body case is peculiar since it does not fit into the conventional time-dependent scattering theory formalism. Several methods are invented to overcome the difficulties caused by the long range behavior of the Coulomb interaction. In the time-dependent framework the formalism of Dollard [1] modifies the Møller wave operator. In stationary approach the method of van Haaringen [2] introduced Coulomb asymptotic states and Mulherin and Zinnes [3] promoted distorted asymptotic states. The connection of the latter two formalism was also investigated [4]. Following the works [5, 6] we will call the asymptotic state of Mulherin and Zinnes as Coulomb-distorted plane wave (CDPW).

The recently introduced surface integral formalism of quantum scattering theory $[5,6,7]$ is valid not only for twobody scattering but it can be extended to scattering of three charged particles. Another advantage of this formalism is that it handles the short and long range interactions on an equal footing. It is said in [6] "All the results ... rely on the asymptotic forms of the plane wave and the Coulomb-distorted plane waves". The CDPW indeed plays an important role in the surface integral formalism. In this framework the full scattering wave function is composed of as a sum of the CDPW and a so called scattered part. Furthermore the surface integral expression of the scattering amplitude also refers to the CDPW.

The explicit three-dimensional form of the CDPW is given by

$$
e^{i \mathbf{k r}}(k r-\mathbf{k r})^{i \gamma} .
$$

Here $\gamma$ is the Sommerfeld parameter and $\mathbf{k}$ is the momentum vector. The bold face letters $\mathbf{k}$ and $\mathbf{r}$ denote elements of the three-dimensional Euclidean space $\mathbb{R}^{3}$, while $k$ and $r$ denote the corresponding magnitudes.

Interestingly the partial wave expansion of the CDPW has been given only recently $[8,9]$ and there is no computer program to evaluate the partial wave components. Here we present an algorithm and FORTRAN-90 code for numerical calculations.

The basic notations are introduced in chapters 2 and 3. Since the CDPW can be expressed by the help of hypergeometric function ${ }_{2} F_{2}(a, a ; a+l+1, a-l ; z)$ we derive an algorithm for the calculation of it in chapter 4 . The numerical results, test runs are presented in chapter 5. Instead of using the direct expression for the CDPW, for two regions, we developed special methods for the calculation. Around the origin we use the Taylor expansion and we determined an upper bound for the error term. Similarly in the asymptotic regions we derived an asymptotic expansion with an estimate for the error term. The detailed mathematical descriptions of the algorithms used for different regions of the complex plane are contained in the Appendix A and B. 


\section{The partial wave expansion of the Coulomb-distorted plane wave}

As we have shown in [9] the partial wave expansion of the Coulomb-distorted plane wave is

$$
e^{i \mathbf{k r}}(k r \mp \mathbf{k r})^{ \pm i \gamma}=\sum_{l=0}^{\infty}(2 l+1) \tau_{l}^{( \pm)}(\gamma, k r) P_{l}(\cos (\vartheta))
$$

where the upper and lower signs correspond to the post and prior form of the CDPW. The angle between the vectors $\mathbf{k}$ and $\mathbf{r}$ is signed by $\vartheta$ and the Legendre polynomial is denoted by $P_{l}(x)$. The radial function $\tau_{l}^{( \pm)}(\gamma, k r)$ can be calculated by the integral

$$
\tau_{l}^{( \pm)}(\gamma, k r)=\frac{1}{2}(k r)^{ \pm i \gamma} \int_{-1}^{1} e^{ \pm i k r x}(1 \mp x)^{ \pm i \gamma} P_{l}(x) d x .
$$

A compact expression for the partial wave component $\tau_{l}^{( \pm)}(\gamma, k r)$ can be given for arbitrary $l$. Using (3) and the formula 2.17.5.6 in [10] we get

$$
\tau_{l}^{(+)}(\gamma, k r)=\frac{(-i \gamma)_{l}}{(1+i \gamma)_{l+1}}(2 k r)^{i \gamma} e_{2}^{i k r} F_{2}(1+i \gamma, 1+i \gamma ; l+2+i \gamma, 1+i \gamma-l ;-2 i k r)
$$

and

$$
\tau_{l}^{(-)}(\gamma, k r)=(-1)^{l} \frac{(i \gamma)_{l}}{(1-i \gamma)_{l+1}}(2 k r)^{-i \gamma} e_{2}^{-i k r}{ }_{2} F_{2}(1-i \gamma, 1-i \gamma ; l+2-i \gamma, 1-i \gamma-l ; 2 i k r),
$$

where $(a)_{n}$ is the Pochhammer symbol and ${ }_{2} F_{2}$ is the generalized hypergeometric function [11].

\section{General description of the program}

The expressions (4) and (5) show that the CDPW can be expressed by the help of the function ${ }_{2} F_{2}(a, a ; a+l+1, a-l ; z)$, where $a=1+i \gamma$ and $a=1-i \gamma$ for the post and prior form of the function. We developed an algorithm for the determination of the hypergeometric function ${ }_{2} F_{2}(a, a ; a+l+1, a-l ; z)$ for almost arbitrary complex $a$ and $z$, some restrictions will be given in the next sections. The calculation of the CDPW can be carried out using the expressions (4) and (5).

The present code uses two freely available FORTRAN-90 codes for the calculation of the Gamma function and for the incomplete Gamma function. The Gamma function is calculated by the CGAMMA code developed by A. H. Morris which can be found in the Naval Surface Warfare Center (NSWC) Library of Mathematics Subroutines. The references $[12,13,14]$ contains the description of the algorithms used by the code CGAMMA. The accuracy of this code is 14 significant decimal digits. The FORTRAN-90 version of this code is due to Alan Miller [15]. The complementer of the incomplete Gamma function is calculated by the code CINCGAM developed by Eric Kostlan and Dmitry Gokhman [16]. Actually we use the FORTAN 90 version of Alan Miller [15]. Arbitrary accuracy can be achieved with the code CINCGAM. Although the $\Gamma(z)$ function can be calculated with the code CINCGAM but in order to increase the speed of the calculation we used the code CGAMMA.

For physical applications there are no large parameters the typical values are $l<20$ and $|a|<100$. We have to deal with however with the cases when the variable $z$ is small or large. In the case of $|z|<1$ we used the Taylor series of the function ${ }_{2} F_{2}(a, a ; a+l+1, a-l ; z)$ and gave an estimate for the remainder if we use only the first $N$ terms of the Taylor series (see Appendix A). In this way we can do arbitrary accuracy calculation in the $|z|<1$ region of the variable $z$. If $|z|$ is large we derived an asymptotic expression for ${ }_{2} F_{2}(a, a ; a+l+1, a-l ; z)$ (see (B.1) and (B.15) in Appendix B) and developed an estimate for the error term.

The code HyP2F2sPEc_v1 in the general case, $(|z| \geq 1$ and $z$ is not in the asymptotic region (see Appendix B)) applies the expression

$$
{ }_{2} F_{2}(a, a ; a+l+1, a-l ; z)=(-z)^{-a} \frac{(a)_{l+1}}{(1-a)_{l}} \sum_{k=0}^{l}\left(\begin{array}{l}
l \\
k
\end{array}\right) \frac{(l+1)_{k}}{k !} \gamma(a+k,-z) z^{-k}
$$

(see: [9]). 


\section{Algorithm of Hyp2F2sPeC_v1}

The code Hyp2F2spec_v1 in the case of $|z| \geq 1$ can be used for $l \geq 0, l$ is integer, $a \in \mathbb{C} \backslash \mathbb{Z}$ and $\operatorname{Re}[a]>0$, in the case of $|z|<1$ it is supposed that $l \geq 0, l$ is integer and $a \in \mathbb{C} \backslash \mathbb{Z}$. The input parameters of the code are $a, l, z, M, E$. The first three correspond to the parameters of the hypergeometric function ${ }_{2} F_{2}(a, a ; a+l+1, a-l ; z)$. The error term of the asymptotic expansion is determined by the integer parameter $M$ such that $M \geq 1$ (see Eq. B15). The absolute error $\epsilon$ is determined by the integer $E(E \geq 0)$ such that $\epsilon=10^{-E}$.

Some non input parameters are determined in the following way. The quantity $n_{f}$ is defined by (A.25), but instead of $n_{f}, n_{f}+1$ will be used in the calculations in order to correct numerical inaccuracy for taking the integer part of a real number. The quantity $q$ is given by the expression (A.10) in the actual calculation we fixed it to the value 0.6. The Appendix A define $\delta_{c}$ and $\delta_{c}^{\prime}$ we take $\delta_{c}=\delta_{c}^{\prime}=0.1$. We have tested numerically the algorithm for different values of $q$ and $\delta_{c}$. It seems that the accuracy and the speed of the code are roughly independent from the value of $q$. The accuracy of the code does not depend on the value of $\delta_{c}$ and the running time of the code has a minimum on the interval $\delta_{c} \in(0,10)$ depending on other parameters.

Algorithm of the numerical calculation

$$
\begin{aligned}
& \text { If }|z|<1 \text { : } \\
& \text { - determine } r_{c}=|z|+\delta_{c} \text { and } r_{c}^{\prime}=r_{c}+\delta_{c}^{\prime} \\
& \text { - calculate } A_{\delta}^{\max } \text { using (A.33) } \\
& \text { - } B_{\delta}^{\max } \text { is given by (A.40) or (A.42) } \\
& \text { - deduce } \chi_{A} \text { and } \chi_{B} \text { using (A.31) and (A.39) respectively } \\
& \text { - } n_{f}^{0} \text { is calculated by (A.25) } \\
& \text { - } n_{f}=n_{f}^{0}+1 \text { in (A.13) } \\
& \text { - determine } N_{0} \text { considering (A.6) and (A.13) } \\
& \text { - calculate }{ }_{2} F_{2}(a, a ; a+l+1, a-l ; z) \text { with the help of expression (A.3) where } n=N_{0}+1 .
\end{aligned}
$$

If $|z| \geq 1$ :

- If $z$ for each $k(0 \leq k \leq l)$ is contained in the asymptotic domain defined by (B.14), furthermore $\epsilon$ greater than the right hand side of the inequality (B.17) then the absolute error satisfies $\left|H_{M}(a, l, z)\right|<\epsilon$. In this case we can use (B.1), (B.3) and (B.15) to calculate ${ }_{2} F_{2}(a, a ; a+l+1, a-l ; z)$.

- In any other case ${ }_{2} F_{2}(a, a ; a+l+1, a-l ; z)$ is determined by (6).

\section{Properties of Hyp2F2spec_v1 and test runs}

We carried out large number of test runs and in this section some properties of the code HyP2F2sPEC_v1 is reviewed. For comparison we used the function HypergeometricPFQ of the software package Mathematica ${ }^{\circledR}$. We denote the absolute value of the difference of the results of our code and the function HypergoemetricPFQ by $D$. As we mentioned earlier due to the use of the CGAMMA function the accuracy can be at most 14 significant decimal digits. In all other modules the accuracy is 32 significant decimal digits. The calculation was carried out on a laptop with processor Intel ${ }^{\circledR}$ Core $^{\mathrm{TM}}$ i5-4200M CPU @ $2.50 \mathrm{GHz}$ (number of cores is 2 and number of threads is 4). In the actual calculations we used the following values for the input parameters $\epsilon=10^{-14}$ and $M=10$. Inside the unit circle we used the Taylor series method described in the Appendix A. Our result showed that the agreement between the codes HyP2F2sPEC_v1 and HypergoemetricPFQ here is the best $D \approx 10^{-32}$. In the asymptotic region we have $D \approx 10^{-17}$ and in the intermediate cases we observed that $D \approx 10^{-16}$.

As for the comparison of the speed of the methods we expected that our code in the asymptotic region is quicker than HypergoemetricPFQ simply due to the reason that the Mathematica ${ }^{\circledR}$ function is a general purpose code. In contrast our Fortran code uses the very special form of the parameters of the hypergeometric function ${ }_{2} F_{2}(a, a ; a+l+1, a-l ; z)$. In the asymptotic region 10 complex points are selected in the following way $z_{n}=$ 


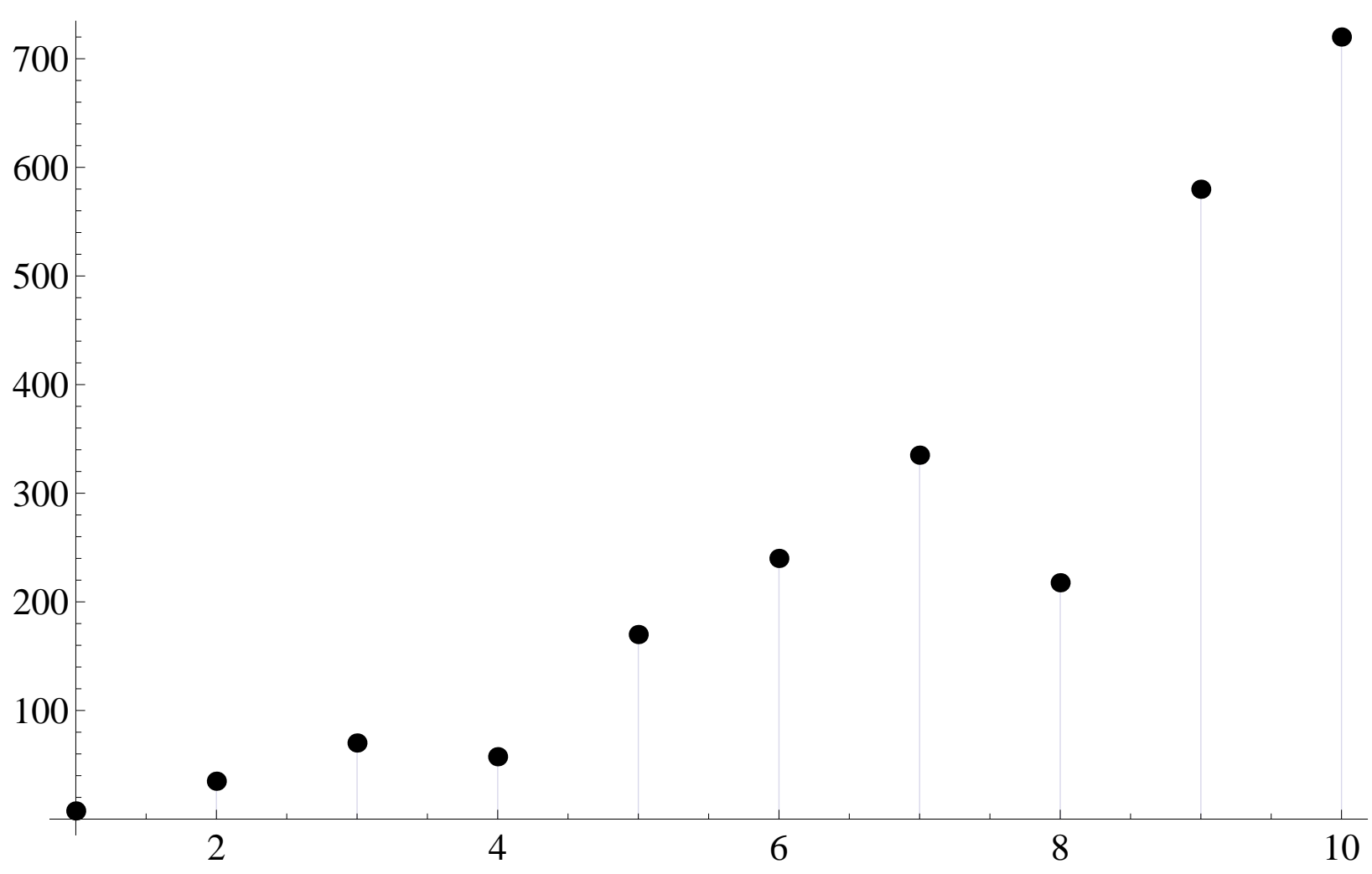

Figure 1. The ratio of the CPU times used by the functions HypergoemetricPFQ and HyP2F2sPEC_v1 for the calculation of the generalized hypergeometric function ${ }_{2} F_{2}(a, a ; a+l+1, a-l ; z)$ at the points $z_{n}=n(-301+183 i)$, where $n=1,2,3, \ldots, 10$. The abscissa shows the value of $n$. For other details see the text. 
$n(-301+183 i)$, where $n=1,2,3, \ldots, 10$. At each points the function values are calculated fifty times and the average value is taken to get an estimate for the CPU time of the calculation at a given $z_{n}$. The Fig. 1 shows the ratio of the CPU times of calculation carried out by the functions HypergoemetricPFQ and HYP2F2sPEC_v1. It can be observed that our function HYP2F2SPEC_v1 significantly quicker than HypergoemetricPFQ. The average CPU time of the selected ten values of $z$ are 0.000468 seconds and 0.08734 seconds for our code and the Mathematica ${ }^{\circledR}$ code respectively.

For checking purposes we give our results at three cases corresponding to the three different regions in $z$. Inside the unit circle if $z=-0.301+0.183 i$ the results is

$$
1.00522217406992826548676481586710-0.01289310297058173780182859374907478 i .
$$

In the asymptotic region using the asymptotic expansion at $z=-301+183 i$ we get

$$
0.255958182470821288862995658127436-0.128153185696130033868227781479859 i \text {. }
$$

In the middle region using the explicit form at $z=-3.01+1.83 i$ the result is

$$
1.02003275291016038616090442223459-0.130277688835653795578119304745202 i \text {. }
$$

\section{Summary}

The calculation of the partial wave components of the CDPW is traced back to the determination of the hypergeometric function ${ }_{2} F_{2}(a, a ; a+l+1, a-l ; z)$. We developed an algorithm and FORTRAN-90 code for the computation of this hypergeomeric function. For the variable $z$ three different regions in the complex plane are considered. In the unit circle we use Taylor series and for large $|z|$ in some cases we apply an asymptotic expansion. In both cases we give error bounds for the neglected terms. For most values of $z$ the code uses an explicit analytic form which is linear combination of incomple Gamma functions. The computer code is based on these mathematical results and the accuracy is 14 significant decimal digits.

\section{Acknowledgments}

This work was supported by U.S. National Science Foundation under Award No. PHY-1415656 and the Hungarian Scientific Research Fund OTKA K112962.

\section{Appendix A. Method for the case $|z|<1$}

In the case $|z|<1$ and $z \in \mathbb{C}$, the code HyP2F2sPEC_v1 can be used if $l \geq 0$ integer and $a \in \mathbb{C} \backslash \mathbb{Z}$. The definition of the generalized hypergeometric function is

$$
{ }_{p} F_{q}\left(a_{1}, \ldots, a_{p} ; b_{1}, \ldots, b_{q} ; z\right) \equiv \sum_{n=0}^{\infty} \frac{\left(a_{1}\right)_{n} \ldots\left(a_{p}\right)_{n}}{\left(b_{1}\right)_{n} \ldots\left(b_{q}\right)_{n}} \frac{z^{n}}{n !}
$$

where $p, q=0,1,2, \ldots ; z, a_{i}, b_{j} \in \mathbb{C} ; i=1, \ldots, p ; j=1, \ldots, q ; b_{j} \neq 0,-1,-2, \ldots$ (see $[10,11,17,18]$ ).

For the sake of the simplicity in our case we use a simpler notation

$$
F_{22}(a, l, z) \equiv{ }_{2} F_{2}(a, a ; a+l+1, a-l ; z) .
$$

The function $F_{22}(a, l, z)$ is analytical on this region so its Taylor series exists. We can write

$$
F_{22}(a, l, z)=\sum_{k=0}^{n} \frac{F_{22}^{(k)}(a, l, z=0)}{k !} z^{k}+R_{n}
$$


where $F_{22}^{(k)}(a, l, z=0)$ is the $k$-th derivative of the function $F_{22}(a, l, z)$ at $z=0$ and the remainder term is $R_{n}$. It is known for $\left|R_{n}\right|$ that

$$
\left|R_{n}\right| \leq \frac{M r_{c}}{r_{c}-|z|}\left(\frac{|z|}{r_{c}}\right)^{n+1}
$$

(see [19]) where $r_{c}=|z|+\delta_{c}$ and $\delta_{c}>0$ is a real number. The $M$ is such a positive real number that the inequality $\left|F_{22}\left(a, l, z_{c}\right)\right| \leq M$ is satisfied for any point $z_{c}$ of the circle $C$ with origin $z=0$ and radius $r_{c}$.

If $M$ is known for a value of $z$ then a natural number $N_{0}$ exists for any $\epsilon>0$ so that the inequality

$$
\frac{M r_{c}}{r_{c}-|z|}\left(\frac{|z|}{r_{c}}\right)^{N+1}<\epsilon
$$

is satisfied for any natural number $N>N_{0}$ because $F_{22}(a, l, z)$ is absolutely convergent and its radius of convergence is infinite. The smallest $N_{0}$ is

$$
N_{0}=\left\lceil\frac{\ln \left[\epsilon\left(1-|z| / r_{c}\right) / M\right]}{\ln \left[|z| / r_{c}\right]}-1\right\rceil
$$

where \lceil\rceil is the ceiling function. In principle, the absolute value of the error can be arbitrary small.

Let us use the notation

$$
f_{i}(z) \equiv \frac{(a)_{i}^{2}}{(a+l+1)_{i}(a-l)_{i}} \frac{z^{i}}{i !}
$$

then $F_{22}(a, l, z)$ is given by

$$
F_{22}(a, l, z)=\sum_{i=0}^{\infty} f_{i}(z)
$$

The ratio test and moreover the expression

$$
q_{n} \equiv\left|\frac{f_{n+1}(z)}{f_{n}(z)}\right| \underset{n \rightarrow \infty}{\longrightarrow} 0
$$

is satisfied so for $|z|<\infty$ and for an arbitrary $0<q<1$ a natural number $n_{f}$ is exist so that the inequality

$$
\left|\frac{f_{n+1}(z)}{f_{n}(z)}\right|<q
$$

is satisfied for any $n \geq n_{f}$ natural number. So we get

$$
\left|F_{22}(a, l, z)\right|=\left|\sum_{i=0}^{\infty} f_{i}(z)\right|<\left|\sum_{i=0}^{n_{f}-1} f_{i}(z)\right|+\frac{\left|f_{n_{f}}\left(z_{c}^{\prime}\right)\right|}{1-q}
$$

if $|z|<\left|z_{c}^{\prime}\right|$. The property $\left|f_{n_{f}+m}\right|<\left|f_{n_{f}}\right| q^{m}, m=1,2, \ldots$ was used. Because of

$$
\left|F_{22}\left(a, l, z_{c}\right)\right|<\sum_{i=0}^{n_{f}-1}\left|f_{i}\left(z_{c}\right)\right|+\frac{\left|f_{n_{f}}\left(z_{c}^{\prime}\right)\right|}{1-q}
$$

we can define $M$ as

$$
M \equiv \sum_{i=0}^{n_{f}-1}\left|\frac{(a)_{i}^{2}}{i !(a+l+1)_{i}(a-l)_{i}}\right| r_{c}{ }^{i}+\frac{\left|\frac{(a)_{n_{f}}^{2}}{n_{n_{f} !(a+l+1)_{n_{f}}(a-l)_{n_{f}}}}\right| r_{c}^{\prime n_{f}}}{1-q}
$$

where $r_{c}<r_{c}^{\prime}=\left|z_{c}^{\prime}\right|=r_{c}+\delta_{c}^{\prime}$ and $\delta_{c}^{\prime}>0$.

Therefore, we can write

$$
q_{n}=\frac{\left|z_{c}^{\prime}\right|}{n+1} \frac{c_{2}^{2}}{c_{1} c_{3}}
$$

for $a, l$ and $z_{c}^{\prime}$ where

$$
c_{1} \equiv \sqrt{(\operatorname{Re}[a]+n-l)^{2}+\operatorname{Im}[a]^{2}},
$$




$$
\begin{gathered}
c_{2} \equiv \sqrt{(\operatorname{Re}[a]+n)^{2}+\operatorname{Im}[a]^{2}}, \\
c_{3} \equiv \sqrt{(\operatorname{Re}[a]+n+l+1)^{2}+\operatorname{Im}[a]^{2}} .
\end{gathered}
$$

The properties $c_{1} \neq 0$ and $c_{3} \neq 0$ is true because of the condition $a \in \mathbb{C} \backslash \mathbb{Z}$. Let us introduce the notation $x \equiv \operatorname{Re}[a]+n$. In our method the expression

$$
\frac{\left|z_{c}^{\prime}\right|}{q} \frac{c_{2}^{2}}{c_{1} c_{3}}-1
$$

will be estimated with three different upper bounds on the next three intervals:

$$
\begin{aligned}
& x \in]-\infty, \chi_{B}[, \\
& x \in\left[\chi_{B}, \chi_{A}[,\right. \\
& x \in\left[\chi_{A},+\infty[\right.
\end{aligned}
$$

and the three upper bounds are

$$
\begin{gathered}
B_{\delta}^{\max } \frac{\left|z_{c}^{\prime}\right|}{q}-1, \\
A_{\delta}^{\max } \frac{\left|z_{c}^{\prime}\right|}{q}-1, \\
2 \frac{\left|z_{c}^{\prime}\right|}{q}-1,
\end{gathered}
$$

respectively. At this moment the values of $\chi_{A}, \chi_{B}, A_{\delta}^{\max }, B_{\delta}^{\max }$ are unknown. Determination of these values will be done later such that the sequence $\left\{B_{\delta}^{\max }, A_{\delta}^{\max }, 2\right\}$ will be monotone decreasing. With this notation it is easy to see that

$$
n_{f}=\left\{\begin{array}{cl}
\left\lceil B_{\delta}^{\max } \frac{\left|z_{c}^{\prime}\right|}{q}-1\right\rceil & \text { if } \operatorname{Re}[a]<\chi_{B}-B_{\delta}^{\max } \frac{\left|z_{c}^{\prime}\right|}{q}+1 \\
\left\lceil\chi_{B}-\operatorname{Re}[a]\right\rceil & \text { if } \chi_{B}-B_{\delta}^{\max } \frac{z_{c}^{\prime} \mid}{q}+1 \leq \operatorname{Re}[a]<\chi_{B}-A_{\delta}^{\max } \frac{\left|z_{c}^{\prime}\right|}{q}+1 \\
\left\lceil A_{\delta}^{\max } \frac{z_{c}^{\prime} \mid}{q}-1\right\rceil & \text { if } \chi_{B}-A_{\delta}^{\max } \frac{z_{c}^{\prime} \mid}{q}+1 \leq \operatorname{Re}[a]<\chi_{A}-A_{\delta}^{\max } \frac{\left|z_{c}^{\prime}\right|}{q}+1 \\
\left\lceil\chi_{A}-\operatorname{Re}[a]\right\rceil & \text { if } \chi_{A}-A_{\delta}^{\max } \frac{z_{c}^{\prime} \mid}{q}+1 \leq \operatorname{Re}[a]<\chi_{A}-2 \frac{\left|z_{c}^{\prime}\right|}{q}+1 \\
\left\lceil 2 \frac{\left|z_{c}^{\prime}\right|}{q}-1\right\rceil & \text { if } \chi_{A}-2 \frac{z_{c}^{\prime} \mid}{q}+1 \leq \operatorname{Re}[a] .
\end{array}\right.
$$

Hereafter we will determinate the upper bounds $B_{\delta}^{\max }$ and $A_{\delta}^{\max }$ of the expression $c_{2}^{2} /\left(c_{1} c_{3}\right)$ and the values of $\chi_{A}$ and $\chi_{B}$.

It is easy to see that

- if $c_{1}<c_{2}$ then $c_{1}<c_{2}<c_{3}$,

- if $c_{1} \geq c_{2}$ and $c_{3}<c_{2}$ then $c_{3}<c_{2} \leq c_{1}$,

- if $c_{1} \geq c_{2}$ and $c_{3} \geq c_{2}$ then $c_{2}^{2} /\left(c_{1} c_{3}\right) \leq 1$,

and that

- $0<c_{1}<c_{2}<c_{3}$ if and only if $l / 2<x$,

- $0<c_{3}<c_{2} \leq c_{1}$ if and only if $x<-(l+1) / 2$,

- $c_{2}^{2} /\left(c_{1} c_{3}\right) \leq 1$ is satisfied if $-(l+1) / 2 \leq x \leq l / 2$. 
First, let us consider the case $x>l / 2$. Let

$$
c_{3}^{\prime} \equiv \sqrt{(x+l)^{2}+\operatorname{Im}[a]^{2}}
$$

and

$$
A(x) \equiv \frac{c_{2}^{2}}{c_{1} c_{3}^{\prime}} .
$$

Then we get the inequality

$$
\frac{c_{2}^{2}}{c_{1} c_{3}}<A(x) \text {. }
$$

The function $A(x)$ is not a constant but it is possible to derive the exact upper bound of $A(x)$. If $l=0$ then $A(x)=1$. We can choose the value 2 as an upper bound of $c_{2}^{2} /\left(c_{1} c_{3}\right)$ in the region $\left[\chi_{A},+\infty\right.$ [ because using this upper bound we get an enough small value of $n_{f}$. The upper bounds have to be valid for all $n \geq n_{f}$ so the sequence $\left\{B_{\delta}^{\max }, A_{\delta}^{\max }, 2\right\}$ has to be monotone decreasing. Easy to see that $A(x)>2$ if and only if $l>0,|\operatorname{Im}[a]|<l / \sqrt{12}$ and $x_{2}^{A}<x<x_{1}^{A}$ where

$$
\begin{aligned}
& x_{1}^{A}=\sqrt{\frac{4}{3} l^{2}-\operatorname{Im}[a]^{2}+\frac{2}{3} l \sqrt{l^{2}-12 \operatorname{Im}[a]^{2}}}, \\
& x_{2}^{A}=\sqrt{\frac{4}{3} l^{2}-\operatorname{Im}[a]^{2}-\frac{2}{3} l \sqrt{l^{2}-12 \operatorname{Im}[a]^{2}}} .
\end{aligned}
$$

In any other case $A(x) \leq 2$. The function $A(x)$ has a local maximum at

$$
x_{\max }^{A}=\sqrt{l^{2}+3 \operatorname{Im}[a]^{2}} .
$$

In the case $x>l / 2$ and $l>0$, the function $A(x)$ is strictly monotone increasing for $x<x_{\max }^{A}$ and strictly monotone decreasing on the other side of $x_{\max }^{A} \cdot \chi_{A}$ will be chosen in such a way that $A(x) \leq 2$ will be satisfied for any $x \geq \chi_{A}$. Considering the numerical inaccuracy our choice is

$$
\chi_{A}= \begin{cases}\left\lceil x_{1}^{A}\right\rceil & \text { if } l>0 \text { and }|\operatorname{Im}[a]|<l / \sqrt{12} \\ \lceil l / 2\rceil & \text { in any other case. }\end{cases}
$$

The fractional part of $a$ reads

$$
\delta \equiv \operatorname{Re}[a]-\lfloor\operatorname{Re}[a]\rfloor
$$

$(\delta \in[0,1[)$ where $\mathrm{L}\rfloor$ is the floor function. For derivation of the exact upper bound of $A(x)$ in the case $l>0$ and $|\operatorname{Im}[a]|<l / \sqrt{12}$ we will use the fact that $x=\lfloor\operatorname{Re}[a]\rfloor+\delta+n,(n=0,1,2, \ldots)$ is a discrete function of the parameter $n$. It is evident that if $\left\lfloor x_{\max }^{A}\right\rfloor+\delta<x_{\max }^{A}$ then $\left\lfloor x_{\max }^{A}\right\rfloor+1+\delta>x_{\max }^{A}$ and if $\left\lfloor x_{\max }^{A}\right\rfloor+\delta>x_{\max }^{A}$ then $\left\lfloor x_{\max }^{A}\right\rfloor-1+\delta<x_{\max }^{A}$. It is also obvious that for any $\delta$ the function $A(x)$ has a maximum at $\left\lfloor x_{\max }^{A}\right\rfloor-1+\delta$ or $\left\lfloor x_{\max }^{A}\right\rfloor+\delta$ or $\left\lfloor x_{\max }^{A}\right\rfloor+1+\delta$ if $x$ exists such that $x_{2}^{A}<x<x_{1}^{A}$. It is uncertain that every such $x$ is grater than $l / 2$ so the determination of the exact upper bound of $A(x)$ is a little bit circumstantial. Therefore, the upper bound $A_{\delta}^{\max }$ of the function $A(x)$ is

$$
A_{\delta}^{\max }= \begin{cases}\max \left\{A\left(\left\lfloor x_{\max }^{A}\right\rfloor+1+\delta\right), 2\right\} & \text { if } f_{A}^{1} \\ \left.\max \left\{A\left(\left\lfloor x_{\max }^{A}\right\rfloor+\delta\right), A\left(\mid x_{\max }^{A}\right\rfloor+1+\delta\right)\right\} & \text { if } f_{A}^{2} \\ \max \left\{A\left(\left\lfloor x_{\max }^{A}\right\rfloor+\delta\right), 2\right\} & \text { if } f_{A}^{3} \\ \left.\max \left\{A\left(\left\lfloor x_{\max }^{A}\right\rfloor-1+\delta\right), A\left(\mid x_{\max }^{A}\right\rfloor+\delta\right)\right\} & \text { if } f_{A}^{4} \\ 2 & \text { in any other case }\end{cases}
$$

where

$$
\begin{aligned}
& f_{A}^{0}: l>0 \text { and }|\operatorname{Im}[a]|<l / \sqrt{12} \\
& f_{A}^{1}: f_{A}^{0} \text { and } \delta \leq x_{2}^{A}-\left\lfloor x_{\max }^{A}\right\rfloor \\
& f_{A}^{2}: f_{A}^{0} \text { and } \delta<x_{\max }^{A}-\left\lfloor x_{\max }^{A}\right\rfloor \text { and } \delta>x_{2}^{A}-\left\lfloor x_{\max }^{A}\right\rfloor \\
& f_{A}^{3}: f_{A}^{0} \text { and } \delta \geq x_{\max }^{A}-x_{\max }^{A} \mid \text { and } \delta \leq x_{2}^{A}+1-\left|x_{\max }^{A}\right| \\
& \left.f_{A}^{4}: f_{A}^{0} \text { and } \delta \geq x_{\max }^{A}-\underset{9}{x_{\max }^{A}}\right] \text { and } \delta>x_{2}^{A}+1-\left[x_{\max }^{A}\right] \text {. }
\end{aligned}
$$


In a similar way $\chi_{B}$ and $B_{\delta}^{\max }$ will be determined when $x<-(l+1) / 2$. In this case

$$
\frac{c_{2}^{2}}{c_{1} c_{3}} \leq \frac{c_{2}}{c_{3}}
$$

because $c_{2} / c_{1} \leq 1$. Let us introduce the notation

$$
B(x) \equiv \frac{c_{2}}{c_{3}} .
$$

We will investigate the expression $B(x)>\Lambda$ where $\Lambda=A_{\delta}^{\max } \geq 2$. The inequality $B(x)>\Lambda$ is satisfied if and only if $|\operatorname{Im}[a]|<\frac{(l+1) \Lambda}{\Lambda^{2}-1}$ and $x_{2}^{B}<x<x_{1}^{B}$ where

$$
\begin{aligned}
& x_{1}^{B}=-\frac{(l+1) \Lambda^{2}}{\Lambda^{2}-1}+\sqrt{\frac{(l+1)^{2} \Lambda^{2}}{\left(\Lambda^{2}-1\right)^{2}}-\operatorname{Im}[a]^{2}}, \\
& x_{2}^{B}=-\frac{(l+1) \Lambda^{2}}{\Lambda^{2}-1}-\sqrt{\frac{(l+1)^{2} \Lambda^{2}}{\left(\Lambda^{2}-1\right)^{2}}-\operatorname{Im}[a]^{2}} .
\end{aligned}
$$

In any other case $B(x) \leq \Lambda . B(x)$ has a local maximum at

$$
x_{\max }^{B}=-\frac{l+1}{2}-\sqrt{\left(\frac{l+1}{2}\right)^{2}+\operatorname{Im}[a]^{2}} .
$$

Moreover, let

$$
\chi_{B}=\left\{\begin{array}{cl}
\left\lceil x_{1}^{B}\right\rceil & \text { if }|\operatorname{Im}[a]|<\frac{(l+1) \Lambda}{\Lambda^{2}-1} \\
\left\lceil-\frac{l+1}{2}\right\rceil & \text { in any other case. }
\end{array}\right.
$$

The upper bound $B_{\delta}^{\max }$ of the function $B(x)$ is

$$
B_{\delta}^{\max }= \begin{cases}\max \left\{B\left(\left\lfloor x_{\max }^{B}\right\rfloor-1+\delta\right), \Lambda\right\} & \text { if } f_{B}^{1} \\ \max \left\{B\left(\left\lfloor x_{\max }^{B}\right\rfloor+\delta\right), B\left(\left\lfloor x_{\max }^{B}\right\rfloor-1+\delta\right)\right\} & \text { if } f_{B}^{2} \\ \max \left\{B\left(\left(x_{\max }^{B}\right\rfloor+\delta\right), B\left(\left\lfloor x_{\max }^{B}\right\rfloor+1+\delta\right)\right\} & \text { if } f_{B}^{3} \\ \max \left\{B\left(\left\lfloor x_{\max }^{B}\right\rfloor+\delta\right), \Lambda\right\} & \text { if } f_{B}^{4} \\ \Lambda & \text { in any other case }\end{cases}
$$

where

$$
\begin{array}{ll}
f_{B}^{0}: & l>0 \text { and }|\operatorname{Im}[a]|<\frac{(l+1) \Lambda}{\Lambda^{2}-1} \\
f_{B}^{1}: & f_{B}^{0} \text { and } \delta \geq x_{1}^{B}-\left\lfloor x_{\max }^{B}\right\rfloor \\
f_{B}^{2}: & f_{B}^{0} \text { and } \delta \geq x_{\max }^{B}-\left\lfloor x_{\max }^{B}\right\rfloor \text { and } \delta<x_{1}^{B}-\left\lfloor x_{\max }^{B}\right\rfloor \\
f_{B}^{3}: & f_{B}^{0} \text { and } \delta<x_{\max }^{B}-\left\lfloor x_{\max }^{B}\right\rfloor \begin{array}{l}
\text { and } \delta<x_{1}^{B}-1-\left\lfloor x_{\max }^{B}\right\rfloor \\
f_{B}^{4}:
\end{array} \quad f_{B}^{0} \text { and } \delta<x_{\max }^{B}-\left\lfloor x_{\max }^{B}\right\rfloor \text { and } \delta \geq x_{1}^{B}-1-\left\lfloor x_{\max }^{B}\right\rfloor .
\end{array}
$$

If $l=0$ then it is possible and worth using the expression

$$
B_{\delta}^{\max }=\max \left\{B\left(\left\lfloor x_{\max }^{B, 0}\right\rfloor-1+\delta\right), B\left(\left\lfloor x_{\max }^{B, 0}\right\rfloor+\delta\right), B\left(\left\lfloor x_{\max }^{B, 0}\right\rfloor+1+\delta\right), 2\right\},
$$

where

$$
x_{\max }^{B, 0}=-\frac{1}{2}-\frac{\sqrt{1+4 \operatorname{Im}[a]^{2}}}{2} .
$$




\section{Appendix B. Method for the case of asymptotic $z$}

The form

$$
{ }_{2} F_{2}(a, a ; a+l+1, a-l ; z)=\frac{(a)_{l+1}}{(1-a)_{l}}(-z)^{-a}\left(\kappa_{l}^{+}(a, z)+e^{z} \kappa_{l}^{-}(a, z)\right)
$$

is derived in [9] and it will be used to get the asymptotic expansion of the function ${ }_{2} F_{2}(a, a ; a+l+1, a-l ; z)$ where

$$
\kappa_{l}^{-}(a, z)=(-1)^{l+1} \sum_{k=0}^{l} \frac{(l+k) !}{k !(l-k) !} \frac{(-1)^{k}}{z^{k}} U(-a+1,-a-k+1,-z)
$$

and

$$
\kappa_{l}^{+}(a, z)=\Gamma(a)_{3} F_{1}(a,-l, l+1 ; 1 ;-1 / z) .
$$

It is easy to calculate the function ${ }_{3} F_{1}(a,-l, l+1 ; 1 ;-1 / z)$ because it is a finite sum. The asymptotic expansion of the confluent hypergeometric function of the second kind is

$$
U(f, g, z)=z^{-f}\left\{\sum_{s=0}^{n-1} \frac{(f)_{s}(1+f-g)_{s}}{s !}(-z)^{-s}+O\left(|z|^{-n}\right)\right\}
$$

(see [11]) where $-3 / 2 \pi<\arg (z)<3 / 2 \pi$. In the work [20], F. W. J. Oliver has given an upper bound for the remainder of the asymptotic expansion of the confluent hypergeometric function of the second kind. We will use this upper bound for the remainder.

In the following Oliver's error bound will be studied and used for our special case. We consider the confluent hypergeometric function of the second kind $U(f, g, z)$ and let $r=|g-2 f|$. We say that $z$ is asymptotic if $z \in$ $\mathbf{R}_{1} \cup \mathbf{R}_{2} \cup \overline{\mathbf{R}}_{2} \cup \mathbf{R}_{3} \cup \overline{\mathbf{R}}_{3}$ (see Figure B.2).

We write $U(f, g, z)$ in the form

$$
U(f, g, z)=z^{-f} \sum_{s=0}^{n-1} \frac{(f)_{s}(f-g+1)_{s}}{s !}(-z)^{-s}+\varepsilon_{n}(z)
$$


(see [11]) where the absolute value of the error is estimated by

$$
\left|\varepsilon_{n}(z)\right| \leq 2 \alpha C_{n}\left|\frac{(f)_{n}(f-g+1)_{n}}{n ! z^{f+n}}\right| \exp \left(\frac{2 \alpha \rho C_{1}}{|z|}\right)
$$

where

$$
C_{n}=\left\{\begin{array}{cl}
1 & , \text { if } z \in \mathbf{R}_{1} \\
\chi(n) & , \text { if } z \in \mathbf{R}_{2} \cup \overline{\mathbf{R}}_{2} \\
\left(\chi(n)+\sigma v^{2} n\right) v^{n} & , \text { if } z \in \mathbf{R}_{3} \cup \overline{\mathbf{R}}_{3}
\end{array}\right.
$$

and

$$
\begin{gathered}
\sigma=\left|\frac{g-2 f}{z}\right|, \\
v=\left(\frac{1}{2}+\frac{1}{2} \sqrt{1-4 \sigma^{2}}\right)^{-\frac{1}{2}}, \\
\chi(n)=\sqrt{\pi} \Gamma\left(\frac{1}{2} n+1\right) / \Gamma\left(\frac{1}{2} n+\frac{1}{2}\right) .
\end{gathered}
$$

If $z \in \mathbf{R}_{1} \cup \mathbf{R}_{2} \cup \overline{\mathbf{R}}_{2}$, then

$$
\begin{gathered}
\alpha=\frac{1}{1-\sigma}, \\
\beta=\frac{1-\sigma^{2}+\sigma|z|^{-1}}{2(1-\sigma)}, \\
\rho=\frac{1}{2}\left|2 f^{2}-2 f g+f\right|+\frac{\sigma\left(1+\frac{1}{4} \sigma\right)}{(1-\sigma)^{2}} .
\end{gathered}
$$

If $z \in \mathbf{R}_{3} \cup \overline{\mathbf{R}}_{3}$, then $\sigma$ have to be interchanged by $v \sigma$ and $|z|^{-1}$ by $v|z|^{-1}$ in (B.11), (B.12) and (B.13) (see [21]).

In our special case (B.2) the regions for $U(-a+1,-a-k+1,-z)$ are

$$
\begin{aligned}
\mathbf{R}_{1}= & \{z \in \mathbb{C}: \operatorname{Re}[z] \leq-r\} \\
\mathbf{R}_{2} \cup \overline{\mathbf{R}}_{2}= & \{z \in \mathbb{C}:|\operatorname{Im}[z]| \geq r \text { és } \operatorname{Re}[z] \geq 0\} \cup \\
& \cup\{z \in \mathbb{C}:-r<\operatorname{Re}[z]<0 \text { és }|z| \geq r\} \\
\mathbf{R}_{3} \cup \overline{\mathbf{R}}_{3}= & \{z \in \mathbb{C}:|\operatorname{Im}[z]|<r \text { és } \operatorname{Re}[z] \geq 0 \text { és }|z| \geq 2 r\}
\end{aligned}
$$

where $r=|a-k-1|$. If we choose the same $n(\mathrm{n}=\mathrm{M})$ for all $k$, we can write the functions $U(-a+1,-a-k+1,-z)$ as sums of the same number of terms and we get

$$
\begin{aligned}
\kappa_{l}^{-}(a, z) & =(-1)^{l+1} \sum_{k=0}^{l} \frac{(l+k) !}{k !(l-k) !} \frac{(-1)^{k}}{z^{k}} U(-a+1,-a-k+1,-z) \\
= & \frac{(a)_{l+1}}{(1-a)_{l}}(-1)^{l+1} \sum_{k=0}^{l} \frac{(l+k) !}{k !(l-k) !} e^{z}(-z)^{-k-1} \sum_{s=0}^{M-1} \frac{(1-a)_{s}(k+1)_{s}}{s !} z^{-s}+ \\
& +H_{M}(a, l, z)
\end{aligned}
$$

where the error is

$$
H_{M}(a, l, z) \equiv \frac{(a)_{l+1}}{(1-a)_{l}}(-1)^{l+1} \sum_{k=0}^{l} \frac{(l+k) !}{k !(l-k) !} e^{z}(-z)^{-a-k} \varepsilon_{M}(-z) .
$$

It is easy to see that

$$
\begin{aligned}
\left|H_{M}(a, l, z)\right| \leq & \left|\frac{2(a)_{l+1}(1-a)_{M}}{M !(1-a)_{l}}\right| \sum_{k=0}^{l} \frac{(l+k) !}{k !(l-k) !} e^{\operatorname{Im}[a](\arg (-z)-\arg (z))} \times \\
& \times|z|^{M-k-1} e^{|z| \cos (\arg (z))} E_{M}(z)
\end{aligned}
$$


where

$$
E_{M}(a, l, z) \equiv \alpha C_{M} \exp \left(\frac{2 \alpha \rho C_{1}}{|z|}\right) .
$$

The previous asymptotic form will be used if and only if $z$ is an element of one of the asymptotic regions of (B.14) for each $k(0 \leq k \leq l)$ and if the inequality $\left|H_{M}(a, l, z)\right|<\epsilon$ is satisfied for $\epsilon>0$ and $M$. In any other case we will use the form (6) for the numerical calculation.

\section{References}

[1] J.D. Dollard, J. Math. Phys. 5 (1964) 729-738.

[2] H. van Haaringen, J. Math. Phys. 17 (1976) 995-1000.

[3] D. Mulherin, I.I. Zinnes, J. Math. Phys. 11 (1970) 1402-1408.

[4] R.O. Barrachina, J.J. Macek, J. Math. Phys. 30 (1989) 2581-2584.

[5] A.S. Kadyrov, I. Bray, A.M. Mukhamedzhanov, A.T. Stelbovics, Phys. Rev. A 72 (2005) 032712. 1-7.

[6] A.S. Kadyrov, I. Bray, A.M. Mukhamedzhanov, A.T. Stelbovics, Ann. Phys. (N.Y.) 324 (2009) 1516-1546.

[7] I. Bray, D.V. Fursa, A.S. Kadyrov, A.T. Stelbovics, A.S. Kheifets, A.M. Mukhamdedzhanov, Phys. Rep. 520 (2012) 135-174.

[8] I. Hornyak, A.T. Kruppa, Phys. Rev. A 85 (2012) 022702. 1-8.

[9] I. Hornyak, A.T. Kruppa, J. Math. Phys. 54 (2013) 053502. 1-7.

[10] A.P. Prudnikov, Yu.A. Brychkov, O.I. Marichev, Integrals and Series, Gordon and Breach Science Publishers, New York, 1990.

[11] M. Abramowitz, I.A Stegun (Eds.), Handbook of Mathematical Functions, Dover, New York, 1964.

[12] H. Kuki, Comm. ACM 15 (1972) 262-267.

[13] R. Spira, Math. Comp. 25 (1971) 317-322.

[14] A.R. DiDonato, A.H. Morris, ACM Trans. Math. Software 12 (1986) 377-393.

[15] Alan Miller's Fortran Software, http://jblevins.org/mirror/amiller/

[16] Eric Kostlan, Dmitry Gokhman, http://zeta.math.utsa.edu/ gokhman/ftp/papers/igf.pdf , http://zeta.math.utsa.edu/ gokhman/ftp/code/cdig.f

[17] Y.L. Luke, The special functions and their approximations Vol. I, Academic Press, San Diego, 1969.

[18] L.J. Slater, Generalized Hypergeometric Functions, Cambridge University Press, London, 1966.

[19] W. Rudin, Real and Complex Analysis, McGraw-Hill Book Company, New York, 1987.

[20] F.W.J. Oliver, J. Soc. Indust. Appl. Math. Ser. B Numer. Anal. 2 (1965) 225-243.

[21] NIST Digital Library of Mathematical Functions, http://dlmf .nist.gov/13.7 